\title{
My Lab is on Mars: Geochemical Adventures with the Mars Curiosity Rover
}

\author{
Laurie Leshin ${ }^{1}$ \\ ${ }^{1}$ Worcester Polytechnic Institute, Office of the President, Worcester, MA, USA
}

Since its dramatic and successful landing on August 5, 2012, Mars Curiosity has been exploring Gale Crater seeking evidence that this site hosted a habitable environment in the martian past. The payload aboard Curiosity (Fig. 1) includes both advanced imagers and diverse geochemical and mineralogical instruments tailored to achieve the mission goals:

\section{Cameras}

Mast Camera (Mastcam) is the main science imager with high-resolution stereo and color capability, spectral filters, and ability to capture high definition video.

Mars Hand Lens Imager (MAHLI) is Curiosity's hand lens, and is mounted on the end of the arm. It takes extreme close-up pictures of rocks and soil revealing details at better than $20 \mu \mathrm{m}$ per pixel.

Mars Descent Imager (MARDI) is a color descent imaging system that captured video during Curiosity's landing.

\section{Spectrometers}

Alpha Particle X-Ray Spectrometer (APXS) determines the relative abundances of different elements in rocks and soils and is an improved version of similar instruments flown on Pathfinder, Spirit and Opportunity.

ChemCam uses laser pulses to vaporize material from targets up to $7 \mathrm{~m}$ from the rover. It uses emission spectroscopy to identify the types of atoms excited by the beam, and a telescope to capture detailed images of the sampled rocks and soils.

Chemistry \& Mineralogy X-Ray Diffraction/X-Ray Fluorescence Instrument (CheMin): The first X-ray diffraction instrument flown to another world, it provides measurements of mineralogy of martian samples.

Sample Analysis at Mars (SAM): Includes a gas chromatograph, a quadrupole mass spectrometer and a tunable laser spectrometer with combined capabilities to identify a wide range of carbon-containing compounds and determine the ratios of isotopes of key elements.

\section{Radiation Detectors}

Radiation Assessment Detector (RAD) characterizes Mars' current radiation environment to support future human exploration.

Dynamic Albedo of Neutrons (DAN) senses subsurface H up to $1 \mathrm{~m}$ below the surface.

\section{Environmental Sensors}

Rover Environmental Monitoring Station (REMS) is Curiosity’s weather station, providing data on atmospheric pressure, temperature, humidity, winds, and UV radiation.

In this presentation, I will discuss the geological and geochemical arguments supporting the discovery of a habitable environment, a benign fluvial system recorded in geologic features, with geochemically available key elements and energy sources. In addition to exploring the aqueous system preserved at Gale, geochemical measurements of surface soils have revealed insights into current volatile sources on Mars that could be of use to future human explorers (Fig. 2; [1]). The latest geological and geochemical data from Mars will be reviewed and discussed. 
References:

[1] L Leshin et al. Science DOI: 10.1126/ science.1238937 (2013)

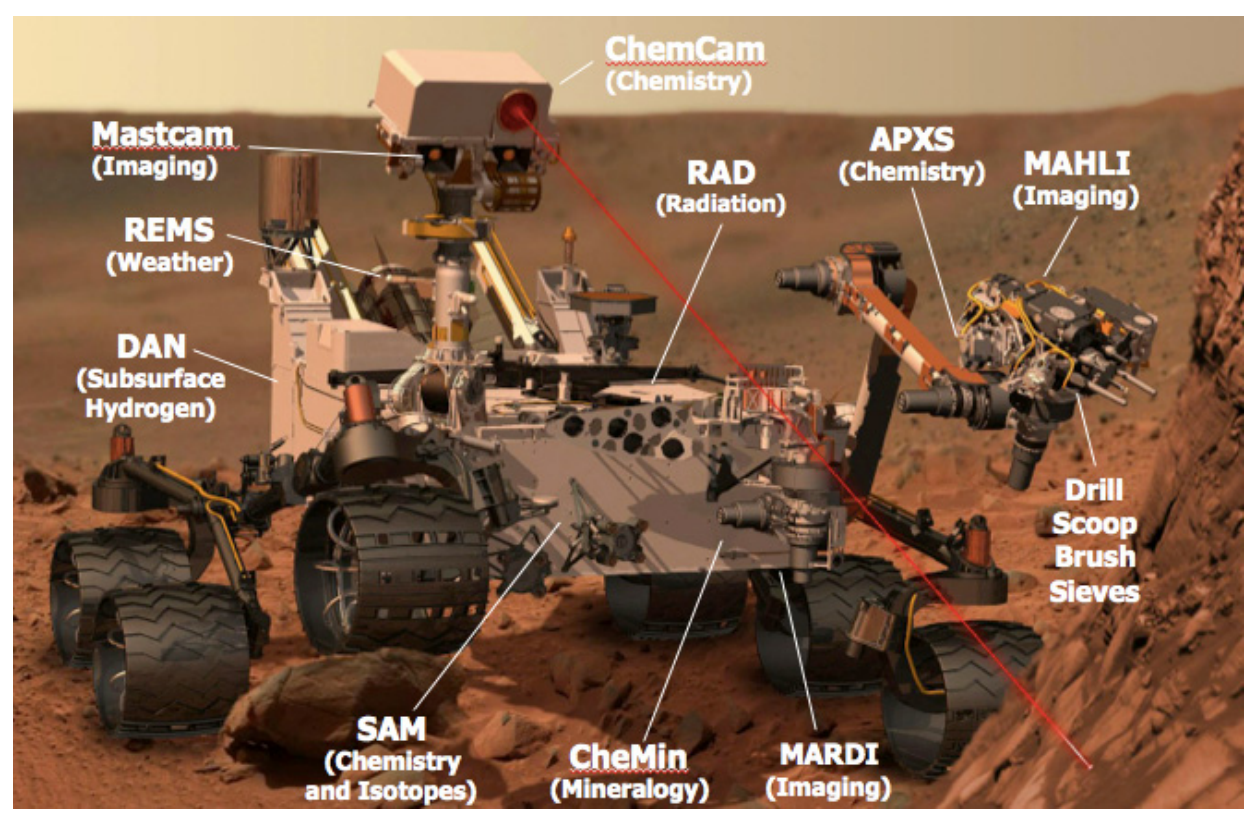

Figure 1. Mars Curiosity Rover Payload (prepared by R. Wiens).

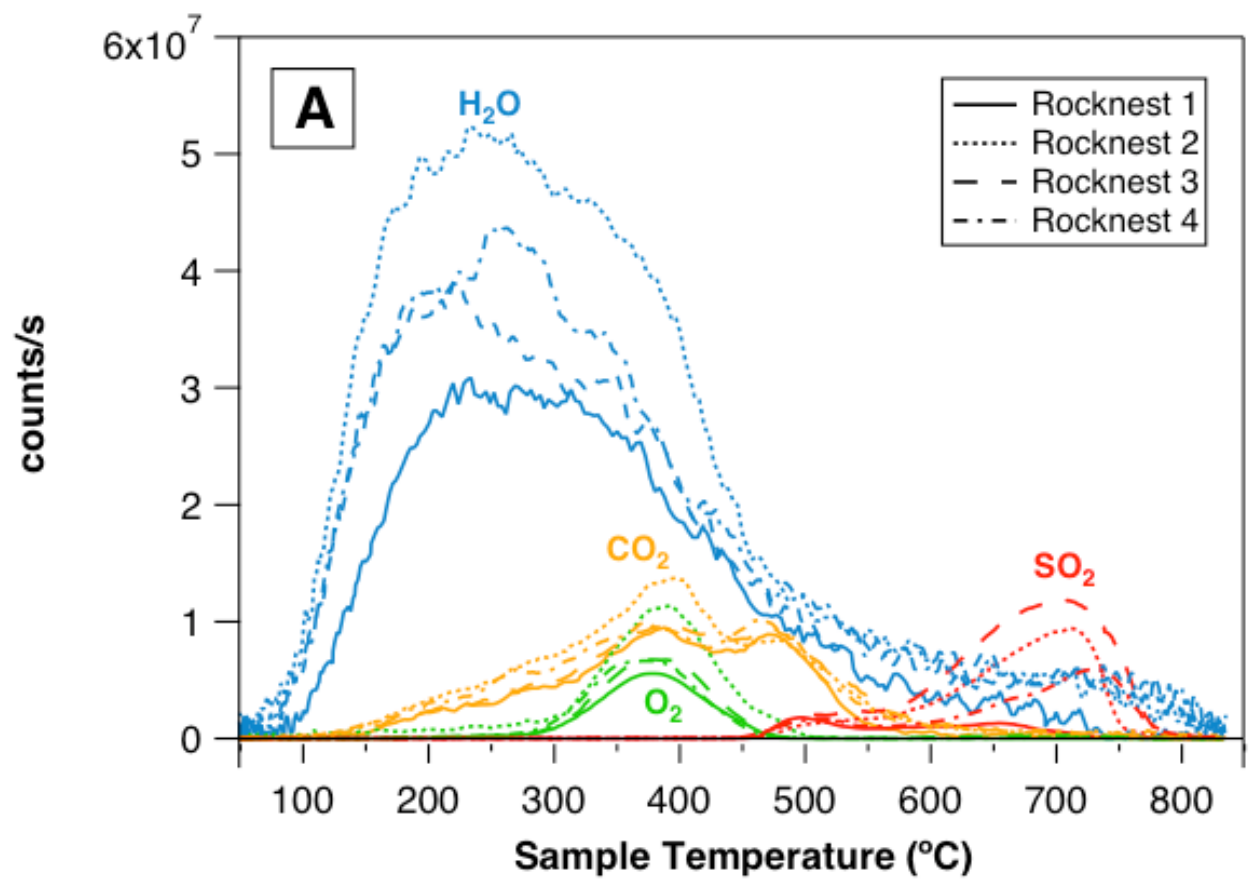

Figure 2. Volatiles released upon heating of Rocknest fines. Water release is equivalent to $\sim 2 \%$ by weight. 